\title{
Structural iconicity in silent gesture
}

\author{
Chuck Bradley (Independent researcher) \\ charles.roger.bradley@gmail.com
}

\begin{abstract}
This study examines structural iconicity in silent gesture by way of the representation and perception of transitive and intransitive events in silent gesture. Specifically, this study focuses on the Iconicity of Quantity and the Iconicity of Complexity, or the strong cross-linguistic tendency for transitive events to be conceptually and structurally more complex than intransitive ones, and for this property to be represented explicitly in verbal morphology where available. Through silent gesture elicitation and perception studies and follow-up handshape analyses, it is demonstrated that non-signers produce and interpret silent gestures in harmony with these iconicity principles. To support our analysis, we identify and recast previous work on event representations in silent gesture and sign language as manifestations of structural iconicity principles.
\end{abstract}

Keywords: silent gesture, structural iconicity, argument structure, event structure

\section{Introduction}

Iconicity is pervasive in language (Perniss, Thompson, and Vigliocco 2010) and connects many different aspects of meaning to form. One of the most obvious and well-studied connections concerns lexical meaning and form. For instance, the sign CAT in American Sign Language and British Sign Language are depictive of a cat's whiskers. However, forms can also be structurally iconic, where meaning and structure have a motivated connection. This has been explored to a limited extent in spoken languages, for instance in the discussion of word or morpheme ordering (Croft 2003; Bybee 1985) and reduplication (Lǐ and Ponsford 2018). Given the limited potential of the oral modality to encode iconicity relative to the visual modality, research into structural iconicity has recently adopted silent gesture paradigms. For instance, one of the most well-researched areas is the representation of constituent ordering patterns in silent gesture, both at the level of the sentence (Hall, Ferreira, and Mayberry 2014; So, Kita, and Goldin-Meadow 2009; Meir et al. 2017, inter alia) and within the nominal domain (Culbertson, Schouwstra, and Kirby 2020; Schouwstra, Kirby, and Culbertson 2017), with the aim of explaining linguistic typology as a partial result of innate cognitive biases. Further, other researchers have probed non-signers' production of grammatical constructions in silent gesture, and comprehension of sign language grammar in perception (e.g., telicity: Strickland et al. 2015; Kuhn et al. 2021; agreement/reference tracking: Schlenker and Chemla 2018; distributivity: Marshall and Morgan 2015; motion events: Schembri, Jones, and Burnham 2005; inter alia).

Despite the volume of work exploring the connection between grammatical meaning and form in sign and silent gesture, there have been few studies that explicitly do so under the banner of structural iconicity. In this way, important connections between the structure of 
spoken and signed languages are missed, namely how structural iconicity may shape communication irrespective of modality. To that end, we contribute new data from silent gesture production and perception experiments, and analyze the results under the lens of structural iconicity with specific reference to the Iconicity of Quantity and the Iconicity of Complexity.

\subsection{Iconicity of Quantity and Complexity}

The principles of Iconicity of Quantity and Iconicity of Complexity state that greater quantities of meaning or more complex meanings correlate with greater quantities of form or more complex forms, respectively (Haspelmath 1993; Croft 2003; Haiman 2008). For example, transitive verbs are cross-linguistically more likely to be more morphologically complex than or derived from intransitive verbs than vice versa (Haspelmath 2015; Ex. 1 a cf. $1 \mathrm{~b}$ ). ${ }^{1}$ This mirrors the modeling of transitives as being more syntactically complex in Generative Grammar (Newmeyer 1992) and cognitive semantics (Jackendoff 1993; Ex. 2).

(1)

$$
\begin{aligned}
& \text { (a) düş- } \emptyset \text {-mek } \\
& \text { 'fall' } \\
& \text { (b) düş-ür-mek } \\
& \text { 'make fall' / 'drop' }
\end{aligned}
$$

Turkish

\section{(2) (a) [Y BECOME/DO Z] ]}

(b) [X CAUSE [Y BECOME/DO Z] ]

Not surprisingly, then, these principles extend to the treatment of classifier constructions in sign languages. Benedicto \& Brentari model transitive classifier constructions as involving two projections, $f_{1} \mathrm{P}$ and $f_{2} \mathrm{P}$, while intransitive classifier constructions involve just one (either of $f_{1} \mathrm{P}$ or $f_{2} \mathrm{P}$; Benedicto and Brentari 2004: Ex. 3). Further, the authors show that this difference in structure has phonological consequences: Transitive and intransitive classifier constructions are differentiated by the valuation of handpart, which specifies the (relative) orientation of the fingertips, palms, backs of the hands, and so on. In intransitive constructions, handpart is a phonological property of the classifier handshape-it is invariant, such that changes in handshape orientation result in ungrammatical or unrelated forms. In transitive constructions, handpart is morphemic and allows greater freedom of (parts of) the hand to be oriented towards different planes.
(a) $[$ f1P $<$ agent $>[\mathrm{VP}]]$
unerg., fixed handpart; p.767, ex. 31a
(b) $[f 2 P<$ theme $>[\mathrm{VP}]]$
unac., fixed handpart; p.767, ex. 31b
(c) $\left[f_{1 P}<\right.$ agent $>\left[f_{2 P}<\right.$ theme $\left.>[\mathrm{VP}]\right]$
trans., handpart variable; p.769, ex. 35a

However, more recent work by Brentari and colleagues (2012; 2017) have since shown that syntactic structure and phonological form of classifier constructions uncouple, such that transitive constructions are more complex by one measure and intransitive constructions are more complex by another. In their studies, the authors asked signers and nonsigners to represent the location, movement (intransitive) or placement (transitive) of two objects (a toy plane and lollipop). They annotated the handshape of each production for

1. In the examples discussed here and throughout, the Iconicity of Quantity and Complexity always make the same predictions, under the assumption that additional structure (quantity) is necessarily more complex (complexity). As such, we continue to use both terms. 
two handshape complexity measures, Finger complexity and Joint complexity (defined below), and measured the distribution of these two features across transitive and intransitive contexts. They observed a strong tendency for signers to use handshapes with more finger complexity when representing intransitive events, and to use handshapes exhibiting more joint complexity when representing transitive events. Further, these tendencies held across sign languages (e.g., American SL, Italian SL, Nicaraguan SL, and so on). These results are counter iconic with respect to the Iconicity of Quantity/Complexity, which again predict that transitive productions should be more complex than intransitive ones. Brentari and colleagues results thus leave open the question of whether these iconicity principles are active in sign languages. Given that Benedicto \& Brentari's analysis was framed around handpart, and Brentari and colleagues analysis centered around handshape complexity, an additional question is what handshape features are relevant to complexity.

Interestingly, though, the non-signers in Brentari and colleagues studies displayed a different pattern: Non-signers used handshapes that were more complex in both Finger and Joint complexity when describing transitive events. These results suggest that non-signers adhere to principles of Iconicity of Quantity and Complexity. However, Brentari and colleagues results may not generalize as, again, they analyzed only three events involving two objects. Further, their analysis only included two handshape features, though more may be relevant. To that end, we contribute new data from silent gesture production, with expanded sets of manual features (6) and event types (72) to confirm whether principles of structural iconicity are present in silent gesture production more generally. Further, it is unknown whether non-signers use principles of structural iconicity in comprehension. To this end, we conducted a silent gesture labeling experiment, hypothesizing that perceivers will also assume that more complexity in visual representation correlates with more underlying structure.

\section{Methods}

We conducted two studies. In the first, we elicited silent gestures from hearing non-signers using video recorded action vignettes. In the second, we elicited text descriptions of the meaning of the silent gestures from non-signers using an online platform.

\subsection{Gesture production study}

Stimuli construction: We produced video recorded vignettes of 72 unique events that involved the manipulation (transitive) or movement (intransitive) of a variety of objects. To verify that the vignettes would be perceived as depicting a transitive or intransitive event, we elicited 25-30 one sentence descriptions of each vignette from 60 participants on Amazon Mechanical Turk. Sentences were annotated for transitivity by the author, such that obligatorily or optionally transitive verbs (e.g., devour and eat, respectively) and ditransitive verbs (e.g., send) were coded as transitive. All other verbs were coded as intransitive. Each vignette elicited at least $80 \%$ agreement in use of transitive or intransitive verb.

Gesture elicitation: Using these action vignettes, we elicited silent gestures from 6 nonsigning participants for a total of $(6 \times 72=) 432$ silent gestures. For this study, we were only interested in how participants manipulated handshape and/or varied the number of hands they used in their gestures, as to make licit comparisons between gesture and classifier constructions. As such, participants were instructed to watch each vignette and then represent 


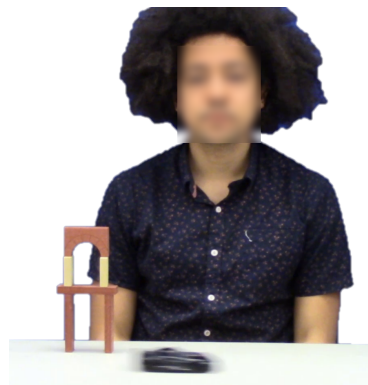

(a)

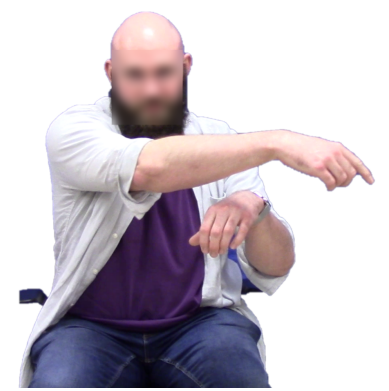

(b)

Figure 1: (a) Still from an action vignette depicting a car passing by a tower. (b) Gesture in response to (a).

the action using only their hands (i.e., speech and full-body gestures were not allowed). Gestures produced in response to a transitive event were coded as transitive, otherwise intransitive. We refer to this as a gesture's inherent transitivity.

Handshape coding: As handshape is related to agentivity in sign languages (e.g., Benedicto and Brentari 2004), we hypothesized that differences between transitive and intransitive gestures could also be captured by handshape. Each gesture was thus coded for handshape, using the coding scheme in Eccarius and Brentari 2008. Each handshape within a production was coded, including the handshape of the non-dominant hand. Coding was performed by the author and one undergraduate researcher separately. Discrepancies between handshape codes were then resolved in tandem. From these handshape codes, the six parameters used in this study could be extracted automatically via a simple Python find-replace routine.

The six parameters are Finger complexity, Joint complexity, Flexion, Flexion of the unselected fingers (USF flexion), Aperture change, and Two-handed. Finger and Joint-complexity concern the number and type of fingers or knuckles involved in a handshape, respectively. Both were scored on an ordinal scale from 1 ('least complex') to 3 ('most complex') following Brentari and colleagues. Flexion describes the degree of curvature of the selected fingers, ranging from 1 ('completely open') to 7 ('completely closed'). USF flexion, in turn, describes the flexion of the unselected fingers, and was ordered along a three-point scale: 1 ('closed'), 0 ('n/a', i.e., all fingers are selected), and -1 ('open'). Finally, Aperture change refers to whether the aperture of the handshape changed from open to closed or vice versa, and Two-handed to whether the production involved two hands. Both were scored categorically ( 1 = 'present'). A more detailed discussion including how these features are individually (and collectively) linked to transitivity in sign languages and silent gesture, see Bradley and Wilbur, submitted.

\subsection{Gesture perception study}

Online study: We conducted the gesture perception study using Amazon Mechanical Turk (AMT). Participants viewed gesture videos and wrote one- to two-sentence descriptions of what they thought the gestures meant. Turkers were required to provide at least one full sentence, so that transitivity of the verb could be established. Further, Turkers were discouraged from providing literal descriptions of the videos (e.g., The person is waving their arms). Each gesture video represented a single trial, and each Turker could choose how many trials to complete. The total number of respondents was 95 , who on average responded to 84 trials 
Finger Complexity

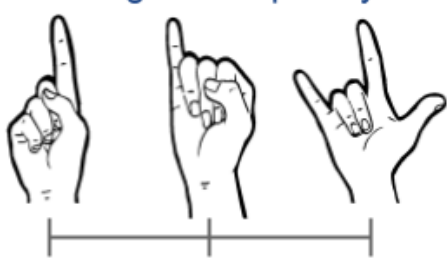

Joint Complexity

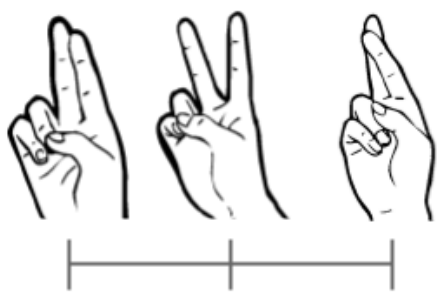

Flexion

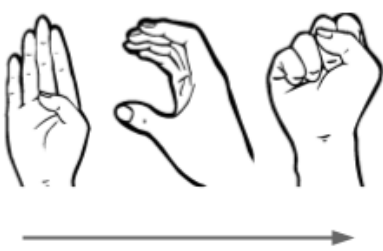

Two-handed?

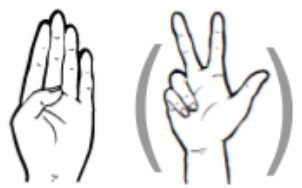

Flexion of unselected fingers

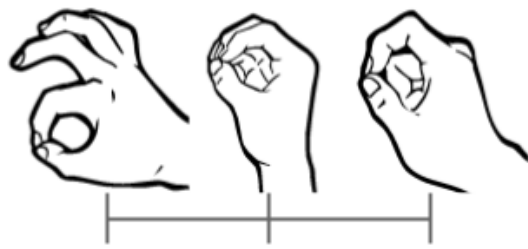

Aperture change

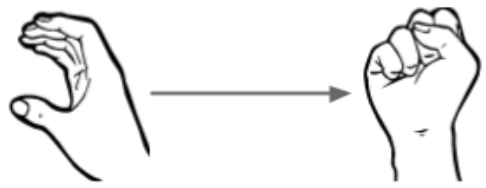

Figure 2: Handshape features included in the studies. Images generated using fonts created by CSLDS, CUHK.

(min: 1; max: 413). Finally, to avoid cheating, an event listener was embedded within each trial, which identified whether the participant played the video. If the event listener was not triggered, that participant's response was discarded and a new Turker provided a replacement response.

Sentence coding: Relevant clauses were coded for transitivity as above with the following exception: Transitive clauses contained a transitive or ditransitive verb and an overt object. For example, I ate a sandwich was considered transitive, but I ate was not. All other clauses were considered intransitive. In case a response contained more than one clause, the average transitivity was taken (e.g., The man dropped the ball and it bounced was coded as 0.5). In some cases, participants included irrelevant clauses, such as It looks like the man is X or It seems like she is Y-ing, in which only the relevant clause was coded for transitivity.

\subsection{Analysis}

For the gesture production study, gestures were labeled as 1 ('transitive') or 0 ('intransitive') according to whether the vignette they depicted was transitive or not. A logit model was then fit to predict a gesture's inherent transitivity from the six handshape features defined above. For the gesture perception study, a gesture's perceived transitivity score was the proportion of transitive sentences it elicited (0-1). An Ordinary Least Squares model was then fit, with handshape features as the independent variables and perceived transitivity score as the dependent variable. If the principles of Quantity and Complexity hold across silent gesture production and perception, then each handshape feature should characterize transitive items (i.e., all $\beta$ 's should be positive). 


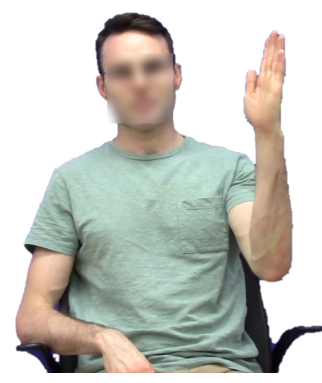

(a)

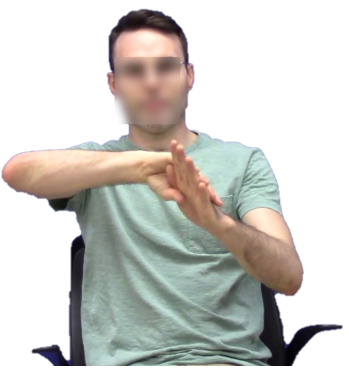

(b)

Figure 3: Gestures depicting (a) the intransitive event, The book fell over, and (b) the transitive event Someone put the book on its side. (b) is more complex than (a) by this analysis: The active hand in (b) is more flexed than in (a), and (b) is two-handed while (a) is one-handed.

\section{Results \& Discussion}

We hypothesized that the principle more form equals more meaning/complexity would explain how non-signers produced and interpreted events with and without an agent in silent gesture. Our hypothesis was confirmed: Both prediction and perception models were significant (production: $\chi^{2}=77.55, \mathrm{p}<0.01$; perception: $\mathrm{F}(6,406)=17.96, \mathrm{p}<0.01$ ). Further, we observed that most features across both the production and perception analyses characterize transitive silent gestures (Tab. 1). That is, gestures representing transitive events were characterized by increased markedness and gestures with increased markedness were more likely to be perceived as transitive. This result is consistent with markedness or complexity being iconically mapped to underlying conceptual structure. That is, there is evidence for the Iconicity of Quantity and Iconicity of Complexity in silent gesture production and perception.

The only feature that marked intransitives was Aperture change. We hypothesized that this feature would be consistent with grasping or releasing an object, and so would also be more associated with transitive gestures. Indeed, all gestures depicting the events crush soda can and drop ball involved aperture change. At the same time, this feature was also used to convey internal movement/change of shape of an object (bowl break, person bend over) or aspectual properties of the event (light turn on, pop balloon), consistent with Wilbur's (2003; 2008) treatment of telic predicates in ASL. Aperture change may thus be more related to aspectual properties of events than to transitivity per se (cf. ditransitive SEND vs. unaccusative WILT in ASL, both involving aperture change).

However, we observed a few discrepancies between previous findings and our results. In the present study, Finger complexity was the only complexity measure to survive statistical analysis, and even then only in production. (The $\beta$ for Joint complexity was near 0 in both analyses). We suspect that the different findings can be attributable to the stimuli used, which we discuss in the following section.

\subsection{Potential effect of stimulus items on results}

The choice of objects may have influenced the results. For instance, while productions depicting a ball dropping were less complex than productions representing dropping a ball, the same might not be true for rakes, paper airplanes, or other objects that have more complex 


\begin{tabular}{lrrrrrr} 
& & & & & \multicolumn{2}{c}{ CI } \\
Feature & $\beta$ & $\mathrm{SE}$ & $\mathrm{z} / \mathrm{t}$ & $\mathrm{p}$ & 0.025 & 0.975 \\
\hline Production & & & & & & \\
Flexion & 0.31 & 0.05 & 6.11 & $<0.01$ & 0.210 & 0.408 \\
NSF flex. & 0.10 & 0.18 & 0.57 & 0.57 & -0.245 & 0.447 \\
Fing. complexity & 0.37 & 0.17 & 2.16 & 0.03 & 0.034 & 0.702 \\
Joint Complexity & -0.01 & 0.19 & -0.07 & 0.94 & -0.387 & 0.359 \\
AAperture & -0.69 & 0.33 & -2.08 & 0.04 & -1.348 & -0.038 \\
Two-handed & 1.12 & 0.26 & 4.61 & $<0.01$ & 0.692 & 1.714 \\
& & & & & & \\
Perception & & & & & & \\
Flexion & 0.05 & 0.01 & 7.66 & $<0.01$ & 0.035 & 0.060 \\
NSF flex. & 0.00 & 0.02 & 0.13 & 0.9 & -0.042 & 0.048 \\
Fing. complexity & 0.03 & 0.02 & 1.5 & 0.14 & -0.010 & 0.077 \\
Joint complexity & -0.00 & 0.03 & -0.09 & 0.93 & -0.052 & 0.047 \\
$\Delta$ Aperture & -0.17 & 0.04 & -3.84 & $<0.01$ & -0.255 & -0.082 \\
Two-handed & 0.19 & 0.03 & 5.65 & $<0.01$ & 0.122 & 0.253
\end{tabular}

Table 1: Model coefficients for Production (Logit) and Perception (OLS) analyses.

shapes. We identified three objects in our dataset-a toy spider (The toy skittered), a paper airplane (The airplane landed), and a clothes hanger (The hanger swung)-that we predicted would elicit more complex handshapes. Each item appeared only in an intransitive context. We trained both the production and perception models excluding these gestures, and tested the models on them. Both models misidentified these intransitive denoting gestures as transitive. The production model predicted that 4 of the 18 gestures $(22 \%)$ were transitive, although none were. The perception model predicted that 10 of the 18 (56\%) were transitive, even though only $5 / 18$ (28\%) were identified by non-signers as transitive. Using the same paradigm, we also examined three transitive events involving objects potentially encoded using low-complexity handshapes, namely a book (Someone put a book on its side), a ball (Someone dropped a ball and Someone bounced a ball). The production model predicted $5 / 18(28 \%)$ of the gestures were transitive, and the perception model predicted that $10 / 18$ (56\%) were transitive. Taken together, these results illustrate some confusability in transitivity determination among objects with relatively intricate or simple shapes.

Additionally, for volumetric objects, like boxes, gesturers did not distinguish between entity and handling handshapes (see Fig. 4). A logit model containing only these gestures was insignificant ( $\chi^{2}=7.77, \mathrm{p}=0.26$ ). Because there was no significant difference in the signal, perceivers at the group level were undecided as to whether these events involved an agent or not $(\mathrm{F}(6,130)=2.51, \mathrm{p}=0.06)$. We note that this issue is not unique to silent gestures, as certain classifier handshapes are homophonous in transitive and intransitive contexts: For instance, the C-handshape can be used to convey a cup moving or an agent moving a cup.

Indeed, Eccarius (2008) notes that the distribution of Finger and Joint complexity across transitive and intransitive classifier constructions holds generally, but that counter-examples can be found. Whether the differences between transitive and intransitive gestures generalizes beyond the specific set of objects used in the present study is an interesting question for future research. It could be the case that handshape complexity reflects real-world 


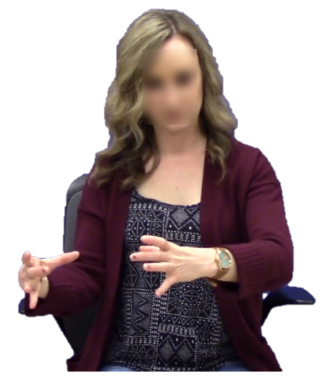

(a)

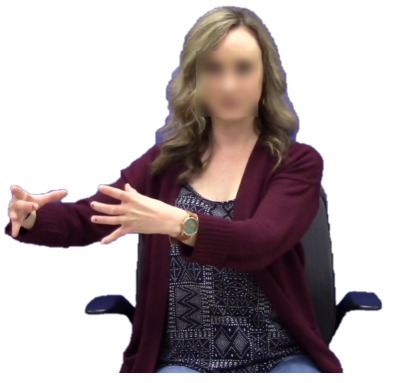

(b)

Figure 4: Gestures depicting (a) The box moved (intrans.) and (b) Someone moved the box (trans.). No obvious handshape features differentiate these two gestures, as both represent the volume of the box.

object complexity. At the same time, though, it has long been reported that handshapes used to depict objects moving are characteristically underspecified in silent gesture (GoldinMeadow, McNeill, and Singleton 1996; Schembri, Jones, and Burnham 2005; Brentari et al. 2012; Brentari et al. 2017). We find support for this in our dataset, where in many instances participants chose to 'draw' the path of a moving object with their index fingers. This tracing strategy was never used to represent an agent moving or manipulating an object.

\subsection{Features relevant to complexity}

In spoken languages, complexity of the sort under investigation here is nearly always the addition of overt morphology. That is, $(1 \mathrm{~b})$ is derived from ( $1 \mathrm{a})$ via the addition of the causative marker $\ddot{u} r$. In the present case, complexity refers to a range of possible handshapes, defined by simultaneously expressed qualities such as flexion and finger complexity. In this sense, it is perhaps less obvious what qualities or features are relevant to complexity in sign language and silent gesture.

As mentioned at the onset, Benedicto and Brentari provide a phonosyntactic account of classifier constructions in ASL, whereby both the syntactic and phonological descriptions of transitive, handling classifier constructions are more complex than intransitve, entity constructions. The complexity in this case is the valuation of handpart: Reiterating from $\$ 1$, it is phonologically specified in entity constructions (i.e., invariant), but morphological in transitive constructions (variant). The difference between these two uses of handpart is the flexibility of handling constructions to be variably oriented. By contrast, Brentari and colleagues show that increased Finger and Joint complexity characterize intransitive and transitive classifier constructions, respectively.

With respect to the present analysis, we showed that Flexion and Two-handed are relevant to non-signers in producing and interpreting transitivity contrasts. Consistent with Brentari and colleagues, Finger complexity was also significantly predictive of transitive productions (we leave unanswered why this feature was not relevant in perception). At the same time, our results are not directly comparable to those obtained in Benedicto and Brentari 2004: We did not ask participants to judge whether particular orientations of intransitivedenoting and transitive-denoting handshapes were acceptable, and we suspect that nonsigners would not have such nuanced (or linguistically informed) judgments-the valuation 
of orientation is thus not a feature we could include in our model. We do note that the examples in Benedicto \& Brentari illustrating the contrast between entity and handling handshapes can be differentiated by Flexion (handling handshapes are more flexed than entity ones), demonstrating at least some consistency between previous and current findings. In all, the expanded stimulus set and handshape feature set used in the present analysis paint a murkier picture of how transitivity contrasts are produced in silent gesture. Further, the results from our perception study suggest that different biases are present when interpreting silent gesture.

\section{Future directions and conclusion}

\subsection{Recasting iconicity}

Structural iconicity has garnered much attention in sign language linguistics, whether explicitly or not, and has been a backgrounded issue in discussions of, e.g., the relationship between sign and gesture (see the Lillo-Martin and Meier 2011 and replies). A well-worked example comes from the discussion of the iconic sources of telicity/boundedness in sign languages: Wilbur $(2003 ; 2008)$ shows that the addition of a gestural boundary, realized as a sharp deceleration towards a point or plane in the signing space, characterizes verbs denoting telic events. Further, Strickland et al. (2015) and Kuhn et al. (2021) show that non-signers are perceptive of this boundary when viewing unfamiliar and artificial signs. In turn, telic predicates are standardly analyzed as involving additional syntactic structure (e.g., Ramchand 2008; Borer 2005). Recasting this collection of facts as a natural consequence of the Iconicity of Quantity/Complexity helps to further link the connection between cognitive abilities (here, detecting perceptual boundaries) and linguistic structure.

Some work on sign languages has indeed fruitfully explored this more is more iconicity directly: Lepic et al. (2016) show that two-handed signs are often used to express notions of plurality, where one vs. more than one is expressed by one hand vs. two hands. Their corpus study surveyed several plural concepts across four geographically and genealogically different sign languages, pointing to conceptual, rather than contact or historical origins to this strategy. Further, this tendency held across different notions of plurality: two-handed signs are used for interaction (e.g., an agent acting on a patient), location (e.g., a figure relative to a ground), and composition (e.g., a pair of wings in ASL sign BUTTERFLY). Further work in this direction may yet reveal other grammatical domains that can be captured by the Iconicity of Quantity/Complexity.

\subsection{Conclusion}

We have advocated for an account that the iconicity principles of Quantity and Complexity, which are predicated on language, hold beyond language. Specifically, previous work on non-signer production and perception across transitivity, telicity, and other grammatical phenomena can be recast with respect to these iconicity principles. However, previous research did not address this kind of iconicity explicitly. What's more, no previous work to our knowledge has examined non-signer production and perception using the same set of stimuli. Our study thus provides more definitive corroborating evidence that non-signers associate more form with more structure in an unfamiliar medium, here silent gesture. Recasting these iconic phenomena as examples structural iconicity allows for the exploration of structure in systems without hard and fast rules, like silent gesture. 


\section{Acknowledgments}

The handshape fonts in Fig. 2 were created by CSLDS, CUHK.

\section{References}

Benedicto, Elena, and Diane Brentari. 2004. "Where did all the arguments go?: Argumentchanging properties of classifiers in ASL." Natural Language \& Linguistic Theory 22 (4): 743-810.

Borer, H. 2005. Structuring Sense Volume 2: The Normal Course of Events. Oxford University Press.

Bradley, C., and R. Wilbur. Submitted. "Visual form and event semantics predict transitivity in silent gestures: Evidence for compositionality.” Cognitive Science, https: / / osf . io / p5ac4/.

Brentari, Diane, Marie Coppola, Pyeong Whan Cho, and Ann Senghas. 2017. "Handshape complexity as a precursor to phonology: variation, emergence, and acquisition." Language Acquisition 24 (4): 283-306.

Brentari, Diane, Marie Coppola, Laura Mazzoni, and Susan Goldin-Meadow. 2012. "When does a system become phonological? Handshape production in gesturers, signers, and homesigners." Natural Language \& Linguistic Theory 30 (1): 1-31.

Bybee, Joan. 1985. "Diagrammatic iconicity in stem-inflection relations.” In Iconicity in Syntax: Proceedings of a symposium on iconicity in syntax, Stanford, June 24-26, 1983, 11. https://doi.org/https://doi.org/10.1075/tsl.6.03byb.

Croft, W. 2003. “Typology.” In Encyclopedia of Cognitive Science, 434-440. London: Nature Publishing Group.

Culbertson, Jennifer, Marieke Schouwstra, and Simon Kirby. 2020. "From the world to word order: deriving biases in noun phrase order from statistical properties of the world." Language 96 (3): 696-717.

Eccarius, Petra. 2008. "A constraint-based account of handshape contrast in sign languages." PhD diss., Purdue University.

Eccarius, Petra, and Diane Brentari. 2008. "Handshape coding made easier: A theoretically based notation for phonological transcription.” Sign Language \& Linguistics 11 (1): 69101.

Goldin-Meadow, S., D. McNeill, and J. Singleton. 1996. "Silence is liberating: removing the handcuffs on grammatical expression in the manual modality." Psychological Review 103 (1): 34 .

Haiman, John. 2008. “In defence of iconicity.” Cognitive Linguistics 19 (1): 35-48.

Hall, Matthew., Victor Ferreira, and Rachel Mayberry. 2014. "Investigating constituent order change with elicited pantomime: A functional account of SVO emergence." Cognitive Science 38 (5): 943-972. 
Haspelmath, Martin. 1993. "More on the typology of inchoative/causative verb alternations." In Causatives and transitivity, edited by Bernard Comrie and Maria Polinsky, 87-120. Amsterdam: John Benjamins.

2015. “Transitivity prominence.” Valency classes in the world's languages 1:131-148.

Jackendoff, Ray. 1993. "The combinatorial structure of thought: The family of causative concepts." In Knowledge and language, edited by E. Reuland and W. Abraham, 31-49. Springer.

Kuhn, Jeremy, Carlo Geraci, Philippe Schlenker, and Brent Strickland. 2021. "Boundaries in space and time: Iconic biases across modalities.” Cognition 210, 104596.

Lepic, Ryan, Carl Börstell, Gal Belsitzman, and Wendy Sandler. 2016. "Taking meaning in hand: iconic motivations in two-handed signs.” Sign Language \& Linguistics 19 (1): 3781.

Lì, Yuèyuán, and Dan Ponsford. 2018. "Predicative reduplication: Functions, their relationships and iconicities.” Linguistic Typology 22 (1): 51-117.

Lillo-Martin, Diane, and Richard P. Meier. 2011. "On the linguistic status of 'agreement' in sign languages.” Theoretical Linguistics 37 (3-4): 95-141. https:/ / doi.org/ doi:10.1515/ thli.2011.009.

Marshall, Chloë R, and Gary Morgan. 2015. "From gesture to sign language: Conventionalization of classifier constructions by adult hearing learners of BSL.” Topics in Cognitive Science 7 (1): 61-80.

Meir, Irit, Mark Aronoff, Carl Börstell, So-One Hwang, Deniz Ilkbasaran, Itamar Kastner, Ryan Lepic, Adi Lifshitz Ben-Basat, Carol Padden, and Wendy Sandler. 2017. "The effect of being human and the basis of grammatical word order: Insights from novel communication systems and young sign languages." Cognition 158:189-207.

Newmeyer, Frederick J. 1992. “Iconicity and generative grammar.” Language, 756-796.

Perniss, Pamela, Robin Thompson, and Gabriella Vigliocco. 2010. "Iconicity as a general property of language: evidence from spoken and signed languages.” Frontiers in Psychology 1:227.

Ramchand, Gillian. 2008. Verb meaning and the lexicon: A first-phase syntax. Cambridge University Press Cambridge.

Schembri, Aadam, Caroline Jones, and Denis Burnham. 2005. "Comparing action gestures and classifier verbs of motion: Evidence from Australian Sign Language, Taiwan Sign Language, and nonsigners' gestures without speech." Journal of Deaf Studies and Deaf Education 10 (3): 272-290.

Schlenker, Philippe, and Emmanuel Chemla. 2018. "Gestural agreement." Natural Language \& Linguistic Theory 36:587-625. https://doi.org/10.1007/s11049-017-9378-8.

Schouwstra, Marieke, Simon Kirby, and Jennifer Culbertson. 2017. "Silent gesture and noun phrase universals." In Proceedings of the 39th Annual Conference of the Cognitive Science Society, 3095-3100.

So, Wing Chee, Sotaro Kita, and Susan Goldin-Meadow. 2009. "Using the hands to identify who does what to whom: Gesture and speech go hand-in-hand." Cognitive Science 33 (1): 115-125. 
Strickland, Brent, Carlo Geraci, Emmanuel Chemla, Philippe Schlenker, Meltem Kelepir, and Roland Pfau. 2015. "Event representations constrain the structure of language: Sign language as a window into universally accessible linguistic biases.” PNAS 112 (19): 59685973.

Wilbur, Ronnie B. 2003. "Representations of telicity in ASL." In Proceedings from the Annual Meeting of the Chicago Linguistic Society, 39:354-368. Chicago Linguistic Society.

2008. "Complex predicates involving events, time and aspect: Is this why sign languages look so similar.” Theoretical issues in sign language research, 217-250. 